\title{
OVERSAMPLED A/D CONVERSION OF NON-BANDLIMITED SIGNALS WITH FINITE RATE OF INNOVATION
}

\author{
Ivana Jovanović, Baltasar Beferull-Lozano \\ Audio-Visual Communications Laboratory \\ Swiss Federal Institute of Technology - EPFL, CH-1015, Lausanne, Switzerland \\ Email: \{Ivana.Jovanovic, Baltasar.Beferull $\} @$ epfl.ch
}

\begin{abstract}
We consider the problem of A/D conversion for non-bandlimited signals that have a finite rate of innovation, in particular, the class of continuous periodic stream of Diracs, characterized by a set of time positions and weights. Previous research has only considered the sampling of these signals, ignoring quantization, which is necessary for any practical application (e.g. UWB, CDMA). In order to achieve accuracy under quantization, we introduce two types of oversampling, namely, oversampling in frequency and oversampling in time. High accuracy is achieved by enforcing the reconstruction to satisfy either three convex sets of constraints related to: 1) sampling kernel, 2) quantization and 3) periodic streams of Diracs which is then said to provide strong consistency or only the first two, providing weak consistency. We propose three reconstruction algorithms, the first two achieving weak consistency and the third one achieving strong consistency. For these three algorithms, respectively, the experimental MSE performance for time positions decreases as $O\left(1 / R_{t}^{2} R_{f}^{3}\right), O\left(1 / R_{t}^{2} R_{f}^{4}\right)$ and $O\left(1 / R_{t}^{2} R_{f}^{5}\right)$, where $R_{t}$ and $R_{f}$ are the oversampling ratios in time and in frequency, respectively. It is also proved theoretically that our reconstruction algorithms satisfying weak consistency achieve an MSE performance of at least $O\left(1 / R_{t}^{2} R_{f}^{3}\right)$.
\end{abstract}

\section{INTRODUCTION}

Recent results in sampling theory [1] have shown that there exist non-bandlimited signals which can be uniformly sampled at a finite rate of innovation (finite number of degrees of freedom per unit time) using an appropriate sampling kernel and then perfectly reconstructed. In all this previous research work, it has been assumed that no quantization of the samples takes place and thus, perfect reconstruction can always be achieved. However, the presence of quantization, which makes perfect reconstruction impossible, is entirely necessary for any practical application involving this type of signals, such as CDMA, UWB [2] and sensor field sampling in sensor networks [3].

In this work, we introduce the presence of quantization and investigate Analog-to-Digital (A/D) conversion of non-bandlimited signals with finite rate of innovation, which has not been considered before. Notice that all previous work in A/D conversion has focused only on bandlimited signals [4]. In this paper, we focus on the A/D conversion of a continuous periodic stream of

This work was supported (in part) by the National Competence Center in Research on Mobile Information and Communications Systems (NCCRMICS), a center supported by the Swiss National Science Foundation.
$K$ Diracs $^{1}$, characterized by a set of time positions $\left\{t_{k}\right\}_{k=0}^{K-1}$ and weights $\left\{c_{k}\right\}_{k=0}^{K-1}$. We study the reconstruction quality under quantization that can be improved by introducing two types of oversampling: a) oversampling in frequency, determined by the bandwidth of the low-pass sampling kernel, and b) oversampling in time, determined by the number of samples in time taken from the acquired filtered signal.

We achieve high accuracy in the reconstruction by enforcing that the reconstructed signal satisfies three convex sets of constraints which are related to: 1) the sampling kernel, 2) the quantization operation itself and 3) the space of continuous periodic streams of $K$ Diracs. A signal reconstruction satisfying the three sets is said to provide strong consistency while if it satisfies only the first two sets it is said to provide weak consistency. We propose two reconstruction algorithms achieving weak consistency and which show experimentally an MSE performance for the time positions ${ }^{2}$ which decreases as $O\left(1 / R_{t}^{2} R_{f}^{3}\right)$ and $O\left(1 / R_{t}^{2} R_{f}^{4}\right)$, where $R_{t}$ and $R_{f}$ are the oversampling ratios in time and frequency, respectively. We prove that the MSE for these algorithms should decrease at least as $O\left(1 / R_{t}^{2} R_{f}^{3}\right)$. We also propose a reconstruction algorithm achieving strong consistency for which we show experimentally an MSE performance $O\left(1 / R_{t}^{2} R_{f}^{5}\right)$.

This paper is organized as follows. Section 2 introduces the class of signals given by continuous-time periodic streams of Diracs. Section 3 defines the concepts of oversampling in time and in frequency. Section 4 introduces weak consistency and strong consistency and proposes three reconstruction algorithms, two of them achieving weak consistency and the other one achieving strong consistency. In Section 5, we prove an upper bound for the MSE performance achieved by weak consistency and in Section 6, we present several experimental results.

\section{SIGNALS WITH FINITE RATE OF INNOVATION}

In [1] it was shown that certain non-bandlimited signals having a finite number of degrees of freedom per unit of time (finite rate of innovation) can be perfectly reconstructed by sampling them at the rate of innovation, using sinc or Gaussian sampling kernel.

In this work, we consider periodic streams of $K$ Diracs, that is, $x(t)=\sum_{k \in \mathbb{Z}} c_{k} \delta\left(t-t_{k}\right)$ with period $\tau$, where $t_{k+K}=t_{k}+\tau$ and $c_{k+K}=c_{k}, \forall k \in \mathbb{Z}$ and $\delta(t)$ denotes a Dirac delta function. This signal has $\frac{2 K}{\tau}$ degrees of freedom per unit of time, since the

\footnotetext{
${ }^{1}$ The extension to other types of signals with finite rate of innovation follows easily from the presented work and it is treated in [5]

${ }^{2}$ The MSE of the weights can be shown to depend strongly on the MSE of the time positions and it is studied in [5]
} 


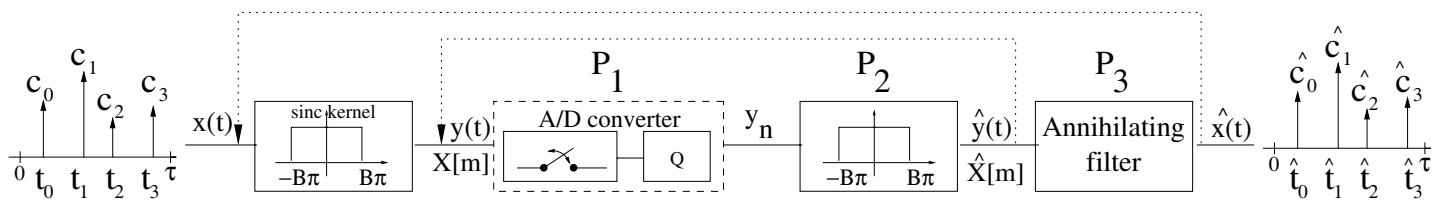

Fig. 1. A/D conversion of a periodic stream of Diracs (example with 4 Diracs).

only knowledge we need to determine the signal uniquely is $K$ time positions $\left\{t_{k}\right\}_{k=0}^{K-1}$ and $K$ weights $\left\{c_{k}\right\}_{k=0}^{K-1}$. This signal can be perfectly reconstructed by first applying a sinc sampling kernel $h_{B}(t)=B \operatorname{sinc}(B t)$ with bandwidth $[-B \pi, B \pi]$, thus obtaining $y(t)=x(t) * h_{B}(t)$, and then taking the $N$ uniform samples $\{y(n T)\}_{n=0}^{N-1}, T=\tau / N$, such that $B \tau=2 M+1 \geq 2 K+1$ and the number of samples is $N \geq 2 M+1$. The periodic stream of $K$ Diracs $x(t)$ can be represented through its Fourier series:

$$
x(t)=\sum_{m \in \mathbb{Z}} X[m] e^{j \frac{2 \pi m}{\tau} \underline{t} t}, \quad X[m]=\frac{1}{\tau} \sum_{k=0}^{K-1} c_{k} e^{-j-\frac{2 \pi m}{\tau} \tau^{-}-\underline{k}} .
$$

The uniform samples of $y(t)$ are given by:

$$
y_{n}=\sum_{m=-M}^{M} X[m] e^{i \underline{2} \underline{\underline{\pi} \underline{m} \underline{m} \underline{\underline{n}} \underline{T}}} \quad \text { where } \quad n=0, \ldots, N-1
$$

Taking at least $2 K+1$ samples $\left\{y_{n}\right\}_{n=1}^{2 K+1}$ we can first directly compute the $2 K+1$ Fourier coefficients $X[\mathrm{~m}]$ of the signal $x(t)$, using a simple DFT. Then, it can be seen from (1) that each exponential term $\left\{u_{k}=e^{-j-\frac{2 \pi t}{\tau} \underline{k}}\right\}_{k=0}^{K-1}$ can be annihilated by a first order FIR filter $A_{k}(z)=\left(1-e^{-j-\frac{2 \pi t}{\tau} \tau_{k}} z^{-1}\right)$. Extension of the filter order to $K$ results in a filter $A(z)=\prod_{k=1}^{K}\left(1-e^{-j \frac{2 \pi t}{\tau} \underline{k}} z^{-1}\right)$ that annihilates the whole signal [1]. In matrix notation, this can be represented as:

$$
\left[\begin{array}{cccc}
X[0] & X[-1] & \ldots & X[-K] \\
X[1] & X[0] & \ldots & X[-(K-1)] \\
\vdots & \vdots & \ddots & \vdots \\
X[K] & X[K-1] & \ldots & X[0]
\end{array}\right]\left(\begin{array}{c}
A[0] \\
A[1] \\
\vdots \\
A[K]
\end{array}\right)=0
$$

where $A[i]$ is the $\mathrm{i}$-th coefficient of the annihilating polynomial. Thus, if we are given the $2 K+1$ exact Fourier coefficients, by setting $A[0]=1$, we can find the unique solution of (3) which gives us all the coefficients of the annihilating filter. The roots of the annihilating filter $A(z),\left\{u_{k}=e^{-j \frac{2 \pi t}{\tau} \underline{k}}\right\}_{k=0}^{K-1}$, reveal the $K$ time positions $\left\{t_{k}\right\}_{k=0}^{K-1}$, while the corresponding weights $\left\{c_{k}\right\}_{k=0}^{K-1}$ can be directly computed from (1).

All previous steps assume no quantization in amplitude and hence, no error in the samples $\left\{y_{n}\right\}_{n=0}^{N-1}$, which ensures the existence of the previous exact solution. In our work we study A/D conversion for these signals and we consider the operation of quantization (see Fig.(1)) performed on the samples $\left\{y_{n}\right\}_{n=0}^{N-1}$. An irreversible loss of information presented by the quantization error in amplitude, introduced in the samples $\left\{y_{n}\right\}_{n=0}^{N-1}$, makes the exact recovery of $x(t)$ no longer possible. As explained in Section 3, oversampling is used to overcome this problem.

\section{OVERSAMPLING IN TIME AND FREQUENCY}

We propose two types of oversampling to compensate the error introduced by quantization. The first one (common method) consists of taking more samples of $y(t)$, so that $N>2 M+1$. This introduces an oversampling in time which is measured by ratio $R_{t}=\bar{n} \bar{M} \bar{M} \overline{1}$.

Notice that we can also perform another additional type of oversampling by extending the bandwidth of the sampling kernel to be greater than the rate of innovation, or equivalently $2 M+1>$ $2 K+1$. We denote this type of oversampling as oversampling in frequency with oversampling ratio $R_{f}=\frac{2 M+1}{2} \bar{K}+\overline{1}$.

Therefore, the number of samples is $N=(2 M+1) R_{t}=$ $(2 K+1) R_{f} R_{t}$, which means that $N$ increases linearly with both oversampling schemes. Both types of oversampling are accuracyenhancing, as it is shown theoretically in Section 5 and experimentally in Section 6.

\section{CONSISTENT RECONSTRUCTION}

In the reconstruction process, we enforce the concept of consistent reconstruction. The idea of consistent reconstruction is to exploit all the knowledge from both the a priori properties of the original signal and the information provided by the quantization process, that is, the quantization bins to which the original samples $\left\{y_{n}\right\}_{n=0}^{N-1}$ belong. Thus, we want to find a reconstruction which is consistent with all the knowledge we have. It is clear that (on average) a consistent reconstruction will provide better reconstruction accuracy than a non-consistent reconstruction. We first define all the properties that a reconstruction should satisfy in order to be consistent. Each property defines a set of signals and requiring satisfaction of a certain property is equivalent to requiring membership in a certain set of signals. The fact that all properties are satisfied by the original signal ensures that the corresponding sets have a nonempty intersection.

The first set of constraints $\boldsymbol{S}_{\mathbf{1}}$ is defined by the quantization operation which is a deterministic operation. The samples $\left\{y_{n}\right\}_{n=0}^{N-1}$ are quantized by a uniform quantizer, that is, $y_{n}^{q}=Q\left(y_{n}\right)=$ $\Delta\left(\left\lfloor y_{n} / \Delta\right\rfloor+1 / 2\right)$ where $\Delta$ is the quantization stepsize. Let $l_{n}=\Delta\lfloor y(t) / \Delta\rfloor$ and $c_{n}=\left[l_{n}, l_{n}+\Delta\right)$. The sequence $\left\{y_{n}^{q}\right\}_{n=0}^{N-1}$ gives us information about the intervals in which all samples lie, namely, $y_{n} \in c_{n}$. The set of these intervals is $N$-dimensional cube and then for $\boldsymbol{y}=\left[y_{0}, \ldots, y_{N-1}\right]^{T}$ and $\boldsymbol{y}^{\boldsymbol{q}}=\mathbb{Q}(\boldsymbol{y})=$ $\left[y_{0}^{q}, \ldots, y_{N-1}^{q}\right]^{T}$ it holds that:

$$
\boldsymbol{y} \in \mathbb{Q}^{-1}\left(\boldsymbol{y}^{\boldsymbol{q}}\right),
$$

as well as all the signals which have samples' amplitudes within the same quantization intervals.

The second set of constraints $\boldsymbol{S}_{\mathbf{2}}$ comes from the observation that the signal $y(t)$ is periodic and bandlimited. Let $\mathcal{V}_{B}$ denote the set of continuous-time periodic signals which are bandlimited to $[-B \pi, B \pi]$. 


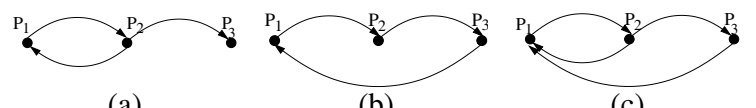

(a)

(b)

(c)

Fig. 2. Algorithms: a)Small-loop; b)Whole-loop; c)Whole \& Small-loop.

By enforcing the previous two sets of constraints we define the concept of weak consistency as follows.

Definition 1 A reconstruction $\hat{x}(t)$ satisfies weak consistency if and only if it is obtained from a $\hat{y}(t)$ that satisfies the following:

1. $\hat{y}(t)$ is a bandlimited signal, $\hat{y}(t) \in \mathcal{V}_{B}$.

2. the samples $\left\{\hat{y}_{n}\right\}_{n=0}^{N-1}$ lie in the same quantization bins as the original ones $\left\{y_{n}\right\}_{n=0}^{N-1}$, or equivalently, $\hat{\boldsymbol{y}} \in \mathbb{Q}^{-1}\left(\boldsymbol{y}^{q}\right)$.

In order to impose weak consistency we define the following two projections.

Projection $\boldsymbol{P}_{\mathbf{1}}$ ([4]) For every estimate $\hat{y}_{n}^{i}, \hat{y}_{n}^{i+1}=\boldsymbol{P}_{\mathbf{1}}\left(\hat{y}_{n}^{i}\right)$ is obtained as:

1. if $\hat{y}_{n}^{i} \in Q^{-1}\left(y_{n}^{q}\right)$ then take $\hat{y}_{n}^{i+1}=\hat{y}_{n}^{i}$.

2. else, take $\hat{y}_{n}^{i+1}$ equal to the bound of the quantization interval $Q^{-1}\left(y_{n}^{q}\right)$ closest to $\hat{y}_{n}^{i}$.

Projection $\boldsymbol{P}_{\mathbf{2}}$ Given an estimate $\hat{y}^{i}(t)$, the new estimate $\hat{y}^{i+1}(t)=$ $\boldsymbol{P}_{\mathbf{2}}\left(\hat{y}^{i}(t)\right)$ is obtained by low-pass filtering $\hat{y}^{i}(t)$, that is $\hat{y}^{i+1}(t)=$ $\hat{y}^{i}(t) * h_{B}(t)$.

Projection $\boldsymbol{P}_{\mathbf{2}}$, which is obtained by applying a pseudoinverse operation, is an orthogonal projection and thus, it is a convex projection, which means that it finds the closest signal in $\mathcal{V}_{B}$. Projection $\boldsymbol{P}_{\mathbf{2}}$ may not always give a reconstruction such that after re-sampling and re-quantization it lies in the same quantization bins as the original one. This is enforced applying projection $\boldsymbol{P}_{\mathbf{1}}$, which is convex. Notice that since $\boldsymbol{S}_{1}$ and $\boldsymbol{S}_{2}$ are convex sets and $\boldsymbol{P}_{\mathbf{1}}$ and $\boldsymbol{P}_{\mathbf{2}}$ are convex projections, the Theorem of alternating projections on convex sets (POCS) [6] can be applied. Starting from the quantized samples $\left\{\hat{y}_{n}^{q}\right\}_{n=0}^{N-1}$, by iterating the projections $\boldsymbol{P}_{1}$ and $\boldsymbol{P}_{2}$, it is ensured that we will reach a reconstruction that belongs to the intersection $\boldsymbol{S}_{\mathbf{1}} \cap \boldsymbol{S}_{\mathbf{2}}$. Since we are interested in reconstructing $\hat{x}(t)$, after finding a reconstruction $\hat{y}(t)$ that satisfies weak consistency, we use the annihilating filter based method explained in Section 2. The process is illustrated in Fig.2(a).

So far, we have not made use of the knowledge about the particular structure of the signal $x(t)$, namely, the fact that $x(t)$ is a periodic stream of $\mathrm{K}$ Diracs. We define our third set of constraints $\boldsymbol{S}_{\mathbf{3}}$ based on this information. Let $\boldsymbol{S}_{\mathbf{3}}$ be defined as a $2 K$ dimensional space $\boldsymbol{S}_{\mathbf{3}}=\left\{\left[\left(t_{0}, c_{0}\right),\left(t_{1}, c_{1}\right), \ldots,\left(t_{K-1}, c_{K-1}\right)\right]^{T}\right.$ : $t_{k} \in[0, \tau), t_{k}<t_{k+1}, c_{k} \in \mathbb{R}$, and $\left.k=0, \ldots, K-1\right\}$ that uniquely determines the signal $x(t)$ and vice versa. It is straightforward to see that this parametric representation of the signal $x(t)$ results in the convex set constraints. This third set $\boldsymbol{S}_{\mathbf{3}}$ is used in order to enforce a stronger sense of consistency, which we call strong consistency and is defined as follows.

Definition 2 A reconstruction $\hat{x}(t)$ satisfies strong consistency if and only if:

1. it satisfies weak consistency

2. $\hat{y}_{n}=\left.h_{b}(t) * \hat{x}(t)\right|_{n T}$ where $\hat{x}(t)$ is a periodic stream of $K$ Diracs.

The concept of strong consistency adds a third property in addition to the two properties defined by weak consistency, that requires estimating of time positions and weights and checking whether the signal $\hat{y}(t)$ can be obtained by filtering a periodic stream of Diracs, namely, $\hat{x}(t)=\sum_{k=0}^{K-1} \hat{c}_{k} \delta\left(t-\hat{t}_{k}\right)$.
From the estimated coefficients $\{\hat{X}(m)\}_{m=-M}^{M}$, we estimate the time positions $\left\{t_{k}\right\}_{k=0}^{K-1}$ using a generalized form of (3) where the left-hand-side matrix has now a size $M \times L$ with $K \leq L \leq M$ and $L$ is the filter order. Notice that here we make use of the oversampling in frequency by making the order of the filter larger than $K$. The solution is found by using the Total Least Squares (TLS) method [6] which can be shown to be equivalent to performing an orthogonal (convex) projection in the vector space of polynomials of order $L$. Thus, in this case the resulting annihilating filter provides $L$ roots and $K$ of them are "correct" roots while the additional $L-K$ roots are created artificially by the method. There are several ways to decide what are the positions of the "correct" roots. We propose two methods:

1. Choose $K$ roots that are closest to the unit circle. This is the common solution used for the retrieval of sinusoids in noise [6]

2. Perform two steps:

(a) Compute roots without increasing the filter order.

(b) Compute roots increasing the filter order and choose the roots that are the closest to the roots in (a).

Notice that by increasing the filter order, extraneous roots can be very close to the unit circle and the first method might fail. The second method does not have this problem and thus, it is the one we use in our experiments.

If there was no quantization and the estimated $\{\hat{X}(m)\}_{m=-M}^{M}$ coefficients were the exact ones then the chosen roots would all lie on the unit circle. However, since there is a quantization error then the next step is to project the obtained roots to the unit circle getting the estimated unit-norm roots $\hat{u}_{k}=e^{-j \frac{2 \pi \hat{t}_{k}}{\tau}}$ and from which we directly estimate the time positions $\left\{\hat{t}_{k}\right\}_{k=0}^{K}$. Then, using (1) we estimate the weights $\left\{\hat{c}_{k}\right\}_{k=0}^{K}$. The whole process including the TLS projection, extracting the correct roots and estimating the time positions and weights, can be seen as the third projection $\boldsymbol{P}_{\mathbf{3}}$.

Projection $\boldsymbol{P}_{\mathbf{3}}$ Given a set of estimated Fourier coefficients $\hat{\boldsymbol{X}}^{i}$, the projection $\boldsymbol{P}_{\mathbf{3}}$ provides $\left\{\left(\hat{t}_{k}^{i+1}, \hat{c}_{k}^{i+1}\right)\right\}_{k=0}^{K-1}$ and a set of Fourier coefficients $\hat{\boldsymbol{X}}^{i+1}$, such that $\hat{X}^{i+1}[m]=\frac{1}{\tau} \sum_{k=0}^{K-1} \hat{c}_{k}^{i+1} e^{-j \frac{2 \pi m \hat{t}_{k}^{i+1}}{\tau}}$

As argued in [5], for large enough $R_{t}$ and $R_{f}$, this projection is convex in the sense that $\hat{\boldsymbol{X}}^{i+1}$ are the closest Fourier coefficients to $\hat{\boldsymbol{X}}^{i}$. Therefore, by applying iterated projections $\boldsymbol{P}_{\mathbf{1}}, \boldsymbol{P}_{\mathbf{2}}$ and $\boldsymbol{P}_{\mathbf{3}}$ on the corresponding convex sets $\boldsymbol{S}_{1}, \boldsymbol{S}_{2}$ and $\boldsymbol{S}_{3}$ we converge to a reconstruction that lies in the intersection $S_{1} \cap S_{2} \cap S_{3}$. We call this algorithm the Whole-loop algorithm (Fig. 2(b)).

As an extension of the Small-loop algorithm we propose a Whole \& Small-loop algorithm (Fig.2(c)) where after reaching weak consistency and obtaining the reconstruction $\hat{x}(t)$, we re-process $\hat{x}(t)$ and re-quantize $\hat{y}(t)$ checking whether its digital representation is exactly the same as the one corresponding to the original signal. This is equivalent to achieving strong consistency. However, because of the order in which the projections are applied, this third algorithm is not guaranteed to converge to a reconstruction satisfying strong consistency (only weak one) and we stop the algorithm after some predefined number of iterations. On the contrary, the convergence of the Small-loop and Whole-loop algorithms is theoretically ensured (POCS), and moreover, numerically speaking, a consistent reconstruction can be approached within a finite number of iterations. 


\section{THEORETICAL PERFORMANCE OF OVERSAMPLING}

Let $\boldsymbol{X}=[X[-M], \ldots, X[M]]^{T}$ and given $\boldsymbol{X}$ and $\hat{\boldsymbol{X}}, M S E(\boldsymbol{X}, \hat{\boldsymbol{X}})=$ $\sum_{m=-M}^{M}|X[m]-\hat{X}[m]|^{2}$. In the next Theorem, we show that the MSE for the weak-sense consistent reconstruction does not depend on the number of Fourier coefficients, or equivalently, on the oversampling in frequency $R_{f}$ while it does depend on the number of samples taken above the Nyquist rate of $y(t)$, which corresponds to the oversampling in time $R_{t}$.

Theorem 1 Given the periodic stream of Diracs $x(t)$ and corresponding $y(t)$ which has $2 M+1(M \geq K)$ Fourier coefficients $\boldsymbol{X}$, there exists an $N_{o}$ such that if $N \geq N_{o}$, there is a constant $c>0$ which depends only on $x(t)$ and not on $R_{t}$ and $R_{f}$, such that for any weak-sense consistent reconstruction $\hat{x}(t)$ with corresponding $\hat{y}(t)$ that has coefficients $\hat{\boldsymbol{X}}$, the following holds:

\section{Proof: see [5].}

$$
\operatorname{MSE}(\boldsymbol{X}, \hat{\boldsymbol{X}}) \leq \frac{c}{R_{t}^{2}} .
$$

This Theorem states that even if we estimate more and more Fourier coefficients (increasing $R_{f}$ ) while keeping $R_{t}$ constant, the upper bound of $M S E(\boldsymbol{X}, \hat{\boldsymbol{X}})$ remains the same. Clearly, since we estimate the time positions from Fourier coefficients, the number of coefficients as well as $M S E(\boldsymbol{X}, \hat{\boldsymbol{X}})$ directly impact the accuracy. Using more Fourier coefficients, which involves increasing $R_{f}$, intuitively improves the time positions estimates. Notice also that $M S E(\boldsymbol{X}, \hat{\boldsymbol{X}})$ will decrease as we increase $R_{t}$ and therefore, $M S E(\boldsymbol{t}, \hat{\boldsymbol{t}})=\sum_{k=0}^{K-1}\left|t_{k}-\hat{t}_{k}\right|^{2}$ will decrease as we increase both $R_{t}$ and $R_{f}$.

Theorem 2 Given $x(t)$, for any reconstruction $\hat{x}(t)$ obtained $u s-$ ing $\boldsymbol{P}_{\mathbf{3}}$ and which satisfies weak consistency, there exist $a \geq 1$ and $b \geq 1$ such that if $R_{t} \geq a$ and $R_{f} \geq b$, there is a constant $c^{\prime}>0$ which depends only on $x(t)$ and not on $R_{t}$ and $R_{f}$, such that the following holds:

$$
M S E(t, \hat{\boldsymbol{t}}) \leq \frac{c^{\prime}}{R_{f}^{3} R_{t}^{2}}
$$

Proof: see [5].

Theorem 2 describes the advantages that both types of oversampling provide for time position estimation. It can be seen that increasing $R_{f}$, we reduce the MSE faster than increasing $R_{t}$.

\section{EXPERIMENTAL RESULTS}

In this section, we show experimental results for the three algorithms illustrated in Fig.(2), with parameters: $K=2, \tau=10$, $t_{k} \in(0, \tau], c_{k} \in[-1,1]$. The positions and the weights are randomly chosen from the corresponding intervals and the results are the average over 100 signals. For the "Small-loop" case, our numerical results confirm Theorems 1 and 2, with a performance of $O\left(1 / R_{t}^{2} R_{f}^{3}\right)$ (Fig.(3) and Fig.(4)). The other two algorithms provide a performance of $O\left(1 / R_{t}^{2} R_{f}^{5}\right)$ (Fig.(5)) and $O\left(1 / R_{t}^{2} R_{f}^{4}\right)$ (Fig.(6)), respectively. A clear outperformance over non-consistent reconstructions (quantization followed by $\boldsymbol{P}_{2}$ and $\boldsymbol{P}_{3}$ ) is observed.

\section{REFERENCES}

[1] M. Vetterli, P. Marziliano, and T. Blu, "Sampling signals with finite rate of innovation," IEEE Trans. on Signal Proc., vol. 50, no. 6, pp. 1417-1428, 2002.

[2] I. Maravić, M. Vetterli, and K Ramchandran, "High-resolution acquisition methods for wideband communication systems," Proc. of ICASSP, 2003.

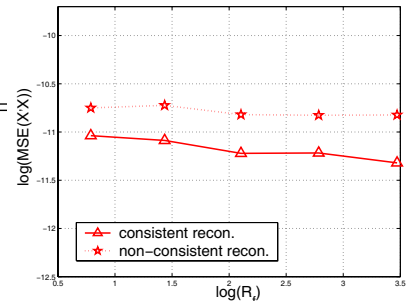

(a)

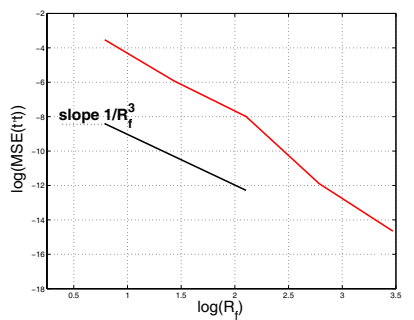

(b)
Fig. 3. Small-loop algorithm. Dependence of accuracy on oversampling in frequency $R_{f}:$ a) MSE of Fourier coefficients; $b$ ) MSE of time positions.

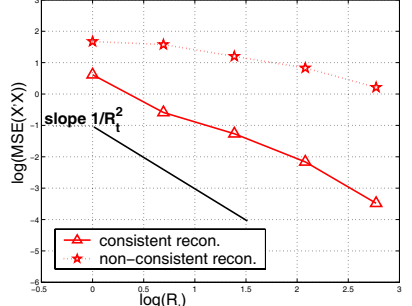

(a)

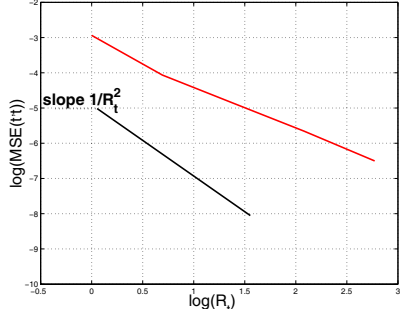

(b)
Fig. 4. Small-loop algorithm. Dependence of accuracy on oversampling in time $R_{t}:$ a) MSE of Fourier coefficients; $b$ ) MSE of time positions.

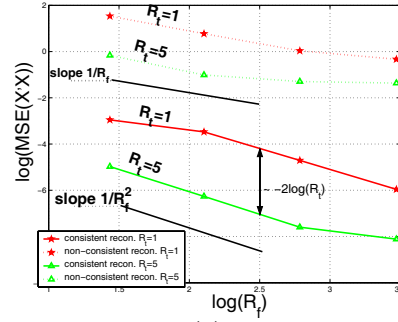

(a)

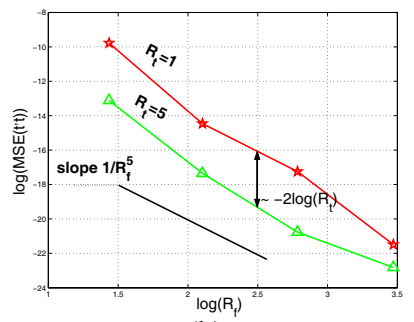

(b)
Fig. 5. Whole-loop algorithm. Dependence of accuracy on oversampling in frequency $R_{f}:$ a) MSE of Fourier coefficients; $b$ ) MSE of time positions.

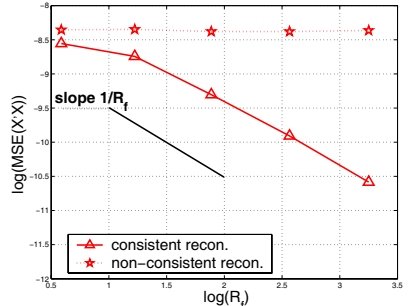

(a)

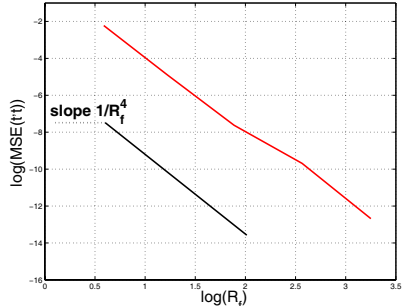

(b)
Fig. 6. Whole \& Small-loop algorithm. Dependence of accuracy on $R_{f}$ : a) MSE of Fourier coefficients; b) MSE of time positions.

[3] P. Ishwar, A. Kumar, and K. Ramchandran, "Distributed sampling for dense sensor networks: a bit-conservation principle," IPSN, 2003.

[4] N. T. Thao and M. Vetterli, "Deterministic analysis of oversampled $A / D$ conversion and decoding improvement based on consistent estimates," Trans. on Signal Proc., vol. 42, no. 3, pp. 519-531, 1994.

[5] I. Jovanović and B. Beferull-Lozano, "Oversampled $A / D$ conversion of non-bandlimited signals with finite rate of innovation," To be submitted to IEEE Trans. on Signal Proc.

[6] T. K. Moon and W. C. Stirling, Mathematical Methods and Algorithms for Signal Processing, Prentice Hall, NJ 07458, 2000. 